\title{
Anisotropic Bianchi Type-III Dark Energy Model with Time- Dependent Deceleration Parameter in Saez-Ballester Theory
}

\author{
Md. Azizur Rahman ${ }^{1}$, M. Ansari ${ }^{2}$ \\ Department of Mathematics, North-Eastern Hill University, Permanent Campus, Shillong-793022, Meghalaya, \\ India.
}

\begin{abstract}
An anisotropic Bianchi type-III cosmological model is investigated in a Saez-Ballester scalar-tensor theory of gravitation. Three different time-dependent skewness parameters along spatial directions are introduced to represent the deviation of pressure from isotropy. To get deterministic solutions of the field equations, we choose variation law of scale factor $S=\left(t^{r} e^{t}\right)^{1 / l}$ which yields a time-dependent deceleration parameter (DP) representing a model that generates a transition of the universe from the early decelerating phase to the present accelerating phase. Some physical and geometrical properties of the model are also discussed.
\end{abstract}

Keywords: Bianchi type-III model, Dark energy, Scalar-tensor theory, Variable deceleration parameter

\section{Introduction}

In recent years, several modifications of Einstein's theory of gravitation have been developed. Among them scalar-tensor theories proposed by Brans and Dicke [1], Saez and Ballester [2], Nordtvedt [3], Wagoner [4], Ross [5], Dunn [6], Barber [7] are important. Saez and Ballester [2] developed a scalar-tensor theory in which the metric is coupled with a dimensionless scalar field in a simple manner. This coupling gives a satisfactory description of the weak fields. Inspite of the dimensionless character of the scalar field, an antigravity regime appears. This theory also suggests a possible way to solve the missing-matter problem in non-flat FRW cosmologies.

Cosmological models in the Saez-Ballester scalar-tensor theory of gravitation have been studied by Singh and Agrawal [8], Ram and Tiwari [9], Singh and Ram [10], Mohanty and Sahu [11], Tripathi et al. [12], Reddy et al. [13], Rao et al. [14], Ram et al. [15] and many. Recently, Naidu et al. [16] obtained Bianchi type-III cosmological model in Saez-Ballester theory considering a variation law for Hubble's parameter which yields a constant value of deceleration parameter. In literature it is common to use a constant deceleration parameter as it duly gives a power law for metric function or corresponding quantity. But for a universe which was decelerating in the past and accelerating at present, the DP must show signature flipping [17-19]. So, in general, the DP is not a constant but time variable. Recently, Amirhashchi et al. [20], Pradhan et al. [21-23] and Yadav [24] investigated Bianchi type cosmological models with time-dependent deceleration parameter.

Motivated by the above works, in the present paper, we have examined an anisotropic Bianchi type-III dark energy model with variable EoS parameter in the framework of Saez-Ballester scalar-tensor theory of gravitation with the help of a variation law of scale factor [20-24] which yields a time-dependent deceleration parameter.

The field equations given by Saez and Ballester [2] for the combined scalar and tensor field

$$
R_{i j}-\frac{1}{2} g_{i j} R-w \emptyset^{n}\left(\emptyset_{, i} \emptyset_{, j}-\frac{1}{2} g_{i j} \emptyset_{, k} \emptyset^{, k}\right)=-T_{i j}^{i}
$$

where $w$ is a dimensionless coupling constant and $n$ is an arbitrary constant.

The scalar field satisfies the equation

It can be easily proved that

$$
2 \emptyset^{n} \emptyset_{; i}^{i}+n \emptyset^{n-1} \emptyset_{, k} \emptyset^{k}=0
$$

$$
T_{; j}^{i j}=0
$$

which are the consequences of the field equations. $T_{i j}^{i j}=0$ is the energy momentum tensor of matter. Here comma and semi-colon designate partial and co-variant differentiation respectively with respect to cosmic time $t$.

\section{The Metric And Field Equations}

We consider the line element for spatially homogeneous and anisotropic Bianchi type-III space-time as

$$
d s^{2}=d t^{2}-A^{2} d x^{2}-e^{-2 \alpha x} B^{2} d y^{2}-C^{2} d z^{2}
$$

where $A, B$ and $C$ are functions of $t$ alone and $\alpha$ is a positive constant. 
The energy momentum tensor of fluid is defined as

$$
T_{i}^{j}=\operatorname{diag}\left[T_{0}^{0}, T_{1}^{1}, T_{2}^{2}, T_{3}^{3}\right]
$$

We can parametrize the energy momentum tensor given in (5) as follows :

$$
\begin{aligned}
T_{i}^{j} & =\operatorname{diag}\left[\rho,-p_{x},-p_{y},-p_{z}\right] \\
& =\operatorname{diag}\left[1,-\omega_{x},-\omega_{y},-\omega_{z}\right] \rho \\
& =\operatorname{diag}[1,-\omega,-(\omega+\gamma),-(\omega+\delta)] \rho
\end{aligned}
$$

where $\rho$ is the energy density of the fluid, $p_{x}, p_{y}, p_{z}$ are the pressures and $\omega_{x}, \omega_{y}, \omega_{z}$ are the directional EoS parameters along $x, y$ and $z$ axes respectively; $\omega(t)=\frac{p}{\rho}$ is the deviation-free EoS parameter of the fluid. The deviation from isotropy is parametrized by setting $\omega_{x}=\omega$ and then introducing skewness parameters $\gamma$ and $\delta$ which are the deviations from $\omega$, respectively along the $y$ and $z$ axes.

The Saez-Ballester field equations (1)-(3) for the metric (4) with the help of (6) lead to the following system of equations

$$
\begin{aligned}
& \frac{\dot{A} \dot{B}}{A B}+\frac{\dot{A} \dot{C}}{A C}+\frac{\dot{B} \dot{C}}{B C}-\frac{\alpha^{2}}{A^{2}}+\frac{w}{2} \emptyset^{n} \dot{\emptyset}^{2}=\rho \\
& \frac{\ddot{B}}{B}+\frac{\ddot{C}}{C}+\frac{\dot{B} \dot{C}}{B C}-\frac{w}{2} \emptyset^{n} \dot{\emptyset}^{2}=-\omega \rho \\
& \frac{\ddot{A}}{\bar{A}}+\frac{\ddot{C}}{C}+\frac{\dot{A} \dot{C}}{A C}-\frac{w}{2} \emptyset^{n} \dot{\emptyset}^{2}=-(\omega+\gamma) \rho \\
& \frac{\ddot{A}}{A}+\frac{\ddot{B}}{B}+\frac{\dot{A} \dot{B}}{A B}-\frac{\alpha^{2}}{A^{2}}-w \emptyset^{n} \dot{\emptyset}^{2}=-(\omega+\delta) \rho \\
& \frac{\dot{A}}{A}-\frac{\dot{B}}{B}=0 \\
& \ddot{\emptyset}+\dot{\emptyset}\left(\frac{\dot{A}}{A}+\frac{\dot{B}}{B}+\frac{\dot{C}}{C}\right)+\frac{n}{2} \frac{\dot{\phi}^{2}}{\emptyset}=0
\end{aligned}
$$

Here, and also in what follows, an overhead dot designates ordinary differentiation with respect to cosmic time $t$. Integrating equation (11), we obtain

$$
B=c_{1} A
$$

where $c_{1}$ is a constant of integration which can be taken as unity without loss of generality so that

$$
B=A
$$

The average scale factor $S$ and the spatial volume $V$ are given by

The generalized mean Hubble's parameter $H$ is defined as

$$
\begin{aligned}
& S=\left(A^{2} C\right)^{\frac{1}{3}} \\
& V=S^{3}=A^{2} C
\end{aligned}
$$

$$
H=\frac{\dot{S}}{S}=\frac{1}{3}\left(H_{1}+H_{2}+H_{3}\right)
$$

where $H_{1}=H_{2}=\frac{\dot{A}}{A}, H_{3}=\frac{\dot{C}}{C}$ are the directional Hubble's parameters in the directions of $x, y$ and $z$ axes respectively.

The deceleration parameter $q$ is defined by

$$
q=-\frac{S \ddot{S}}{\dot{S}^{2}}
$$

The expansion scalar $\theta$, shear scalar $\sigma^{2}$ and the mean anisotropy parameter $A_{m}$ are given by

where $\Delta H_{i}=H_{i}-H(i=1,2,3)$

$$
\begin{gathered}
\theta=u_{; i}^{i}=2 \frac{\dot{A}}{A}+\frac{\dot{C}}{C} \\
\sigma^{2}=\frac{1}{2} \sigma_{i j} \sigma^{i j}=\frac{1}{3}\left(\frac{\dot{A}}{A}-\frac{\dot{C}}{C}\right)^{2} \\
A_{m}=\frac{1}{3} \sum\left(\frac{\Delta H_{i}}{H}\right)^{2}
\end{gathered}
$$




\section{Solutions And The Model}

The field equations (7)-(10) and (12) are a system of five independent equations with seven unknown parameters $A=B, C, \rho, \omega, \gamma, \delta$ and $\emptyset$. Hence two additional constraints are required to obtain explicit solutions of these field equations.

Since the metric (4) is completely characterized by average scale factor therefore we consider following Amirhashchi et al. [20], Pradhan et al. [21-23] and Yadav [24] that average scale factor is an increasing function of time as following:

where $r$ and $l$ are positive constants.

$$
S=\left(t^{r} e^{t}\right)^{\frac{1}{l}}
$$

We also assume that the shear scalar $\sigma$ is proportional to the expansion scalar $\theta$ which gives

$$
A=C^{m}
$$

where $m$ is a constant. The motive behind assuming this condition is explained with reference to Thorne (1967) [25], the observations of the velocity red-shift relation for extragalactic sources suggest that Hubble expansion of the universe is isotropy today within $\approx 30$ percent (Kantowski and Sachs [26]; Kristian and Sachs [27]). To put more precisely, red-shift studies place the limit

$$
\frac{\sigma}{H} \leq 0.3
$$

on the ratio of shear $\sigma$ to Hubble constant $H$ in the neighbourhood of our galaxy today. Collin et al. (1980) [28] have pointed out that for spatially homogeneous metric, the normal congruence to the homogeneous expansion satisfies that the condition $\frac{\sigma}{\theta}$ is constant.

Solving the field equations (7)-(10) with the help of (15), (22) and (23), we obtain

Hence the metric (4) of the universe takes the form

$$
\begin{aligned}
A=B & =\left(t^{r} e^{t}\right)^{\frac{3 m}{l(2 m+1)}} \\
C & =\left(t^{r} e^{t}\right)^{\frac{3}{l(2 m+1)}}
\end{aligned}
$$

$$
d s^{2}=d t^{2}-\left(t^{r} e^{t}\right)^{\frac{6 m}{l(2 m+1)}} d x^{2}-e^{-2 \alpha x}\left(t^{r} e^{t}\right)^{\frac{6 m}{l(2 m+1)}} d y^{2}-\left(t^{r} e^{t}\right)^{\frac{6}{l(2 m+1)}} d z^{2}
$$

\section{Physical And Geometrical Properties Of The Model}

Equation (26) represents Bianchi type-III dark energy model in Saez-Ballester theory of gravitation. At $t=0$, all the spatial scale factors are zero which implies that the derived model has a point-type singularity at $t=0$ [29].

Equation (22) with the help of equation (18) yields a time-dependent deceleration parameter as

$$
q=\frac{l r}{(t+r)^{2}}-1
$$

We observe that $q>0$ for $t<\sqrt{l r}-r$ and $q<0$ for $t>\sqrt{l r}-r$. For $l \geq 3$ and $r=1$, our model evolves from decelerating phase to accelerating phase. Recent observations of type-Ia supernovae [30, 31] suggests that the present universe is accelerating and the value of DP lies in the range $-1<q<0$. So, our derived model is consistent with the recent observations.

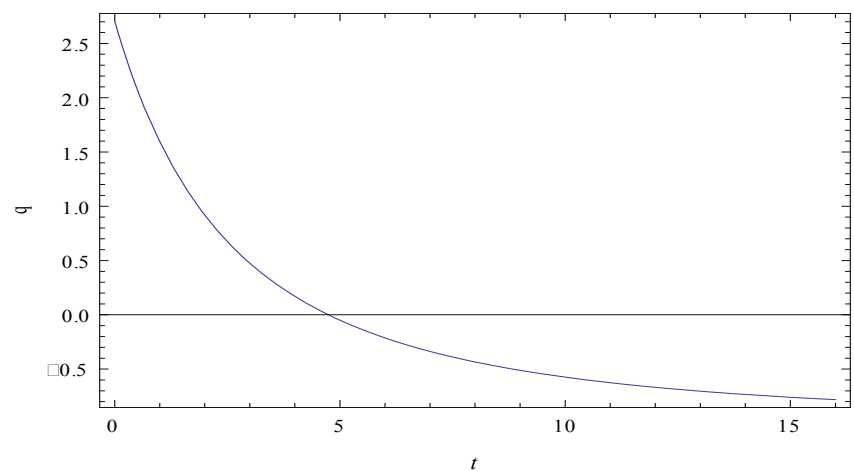

Fig. 1 : The plot of DP (q) versus time (t)

The spatial volume

Hubble's parameter

$$
V=\left(t^{r} e^{t}\right)^{\frac{3}{l}}
$$


The expansion scalar

$$
H=\frac{1}{l}\left(1+\frac{r}{t}\right)
$$

$$
\theta=\frac{3}{l}\left(1+\frac{r}{t}\right)
$$

Shear scalar

$$
\sigma^{2}=\frac{3(m-1)^{2}}{l^{2}(2 m+1)^{2}}\left(1+\frac{r}{t}\right)^{2}
$$

The mean anisotropy parameter

$$
A_{m}=\frac{1}{3}\left[\frac{9\left(2 m^{2}+1\right)}{(2 m+1)^{2}}+1\right]
$$

Also, we have

$$
\frac{\sigma^{2}}{\theta^{2}}=\frac{(m-1)^{2}}{3(2 m+1)^{2}} \neq 0, \quad m>1
$$

The scalar field

$$
\varnothing=\left[\emptyset_{0}\left(\frac{n+2}{2}\right) \int \frac{d t}{\left(t^{r} e^{t}\right)^{\frac{3}{l}}}\right]^{\frac{2}{n+2}}
$$

In the derived model (26), the present value of DP is estimated as

$$
q_{0}=-1+\frac{r}{l H_{0}^{2} t_{0}^{2}}
$$

where $H_{0}$ is the present value of Hubble's parameter and $t_{0}$ is the age of the universe at present epoch. If we set $l=3$ and $r=1$ in Eq. (35), we obtain $q_{0}=-0.67$ which matches the observed value of the DP at the present epoch [32].

The energy density

The EoS parameter

$$
\rho=\frac{9 m(m+2)}{l^{2}(2 m+1)^{2}}\left(1+\frac{r}{t}\right)^{2}-\frac{\alpha^{2}}{\left(t^{r} e^{t}\right)^{\frac{6 m}{l(2 m+1)}}}+\frac{w \emptyset_{0}^{2}}{2\left(t^{r} e^{t}\right)^{\frac{6}{l}}}
$$

Skewness parameters

$$
\omega=\frac{1}{\rho}\left[\frac{w \emptyset_{0}^{2}}{2\left(t^{r} e^{t}\right)^{\frac{6}{l}}}-\frac{9\left(m^{2}+m+1\right)}{l^{2}(2 m+1)^{2}}\left(1+\frac{r}{t}\right)^{2}+\frac{3(m+1)}{l(2 m+1)} \frac{r}{t^{2}}\right]
$$

and

$$
\gamma=0
$$

$$
\delta=\frac{1}{\rho}\left[\frac{w \emptyset_{0}^{2}}{2\left(t^{r} e^{t}\right)^{\frac{6}{l}}}-\frac{9\left(2 m^{2}-m-1\right)}{l^{2}(2 m+1)^{2}}\left(1+\frac{r}{t}\right)^{2}+\frac{3(m-1)}{l(2 m+1)} \frac{r}{t^{2}}+\frac{\alpha^{2}}{\left(t^{r} e^{t}\right)^{\frac{6 m}{l(2 m+1)}}}\right]
$$

From the above results, we observe that at $t=0$, the spatial volume is zero and other parameters $\theta, \sigma, H$ diverge. This implies that the universe starts evolving with zero volume at $t=0$ and expands with cosmic time $t$ which is a big bang scenario. At $t \rightarrow \infty$, we get $q=-1$ and $\frac{d H}{d t}=0$, which implies the greatest value of Hubble's parameter and fastest rate of expansion of the universe. The negative value of $q$ would accelerate and increase the age of the universe. Figure 1 depicts the dynamics of DP versus cosmic time $t$. It can be seen that initially the value of DP was positive and its value becomes negative later on. This clearly explains that the universe had a decelerated expansion in the past and has an accelerating expansion at present as observed by the recent observations of SN Ia. So, the derived model is fit to describe the dynamics of the late time evolution of the universe.

Since $\frac{\sigma^{2}}{\theta^{2}} \neq 0$ except at $m=1$, hence the model is anisotropic for all values of $m$ except for $m=1$. The mean anisotropy parameter is uniform throughout the evolution of the universe.

It is important to note here that $\lim _{t \rightarrow 0}\left(\frac{\rho}{\theta^{2}}\right)$ turn out to be constant. Therefore the model approaches homogeneity and matter is dynamically negligible near the origin. This is in good agreement with the result already given by Collins [33].

The scalar field is found to be a decreasing function of time. At $t=0$, it becomes infinite and for large value of time it vanishes.

The energy density $\rho$ and EoS parameter $\omega$ are both decreasing functions of time and they become constant at large values of $t$. From Eqs. (36) and (37), we observe that EoS parameter $\omega$ is time-dependent, it 
can be a function of redshift $z$ or scale factor $S$ as well. So, if the present work is compared with experimental results [34-37], then one can find the limit of $\omega$ provided by Eq. (37) within acceptable range.

For different choices of $l$ and $r$, we can obtain a class of viable DE models in Bianchi type-III space-time and such models are consistent with recent observations.

\section{Conclusion}

An anisotropic Bianchi type-III dark energy cosmological model is investigated in the framework of Saez-Ballester scalar-tensor theory of gravitation. Exact solutions of the field equations are obtained by assuming suitable physical conditions that yield a time-dependent DP representing a model which generates a transition from early decelerating phase to the present accelerating phase of the universe. The EoS parameter and skewness parameters appear to be time-dependent. The derived model can be a viable cosmological model to explain the present acceleration of the universe.

\section{References}

[1]. C. H. Brans., R. H. Dicke, Mach's principle and a relativistic theory of gravitation, Physical Review, 124(3), 1961, 925-935

[2]. D. Saez, V. J. Ballester, A simple coupling with cosmological implications, Physics Letters, A 113 (9), 1985, 467-470

[3]. K. Nordtvedt, Post-Newtonian meric for a general class of scalar-tensor gravitational theories and observational consequences, Astrophysical Journal, 161, 1970, 1059-1067

[4]. R. V. Wagoner, Scalar-tensor theory and gravitational waves, Physical Review D 1, 1970, 3209-3216

[5]. D. K. Ross, Scalar-tensor theory of gravitation, Physical Review D 5, 1972, 284-290

[6]. K. A. Dunn, A scalar-tensor theory of gravitation, Journal Mathematical Physics, 15, 1974, 2229-2231

[7]. G. A. Barber, On two self-creation cosmologies, General Relativity and Gravitation, 14 (2), 1985,117

[8]. T. Singh, A. K. Agrawal, Some Bianchi type cosmological models in a new scalar-tensor theory, Astrophysics Space Science, 182 (2), 1991, 289-312

[9]. S. Ram, S. K. Tiwari, Inhomogeneous plane-symmetric models in a scalar-tensor theory, Astrophysics Space Science, 259 (1), 1998, 91-97

[10]. C. P., Singh, S. Ram, Unified description of early universe in scalar-tensor theory, Astrophysics Space Science, 284 (4), 2003 , $1199-1206$

[11]. G. Mohanty, S. K. Sahu, Bianchi $\mathrm{VI}_{0}$ cosmological model in Saez and Ballester theory, Astrophysics Space Science, 288, 2003, 611-618

[12]. S. K. Tripathi, S. K. Nayak, S. K. Sahu, T. R. Routray, Massive string cloud cosmologies in Saez-Ballester theory of gravitation, International Journal of Theoretical Physics, 48, 2009, 213-225

[13]. D. R. K. Reddy, R. L. Naidu., V. U. M. Rao, Axially symmetric cosmic strings in a scalar-tensor theory, Astrophysics Space Science, 306, 2006, 185-188

[14]. V. U. M. Rao, G. Sreedevikumari, K. V. S. Sireesha, Anisoropic universe with cosmic string and bulk viscosity in a scalar-tensor theory of gravitation, Astrophysics Space Science, 335, 2011, 635-638

[15]. S. Ram, M. Zeyauddin, C. P. Singh, Bianchi type-V cosmological models with perfect fluid and heat flow in Saez-Ballester theory, Pramana-Journal of Physics, 72, 2009, 415-427

[16]. R. L. Naidu, B. Satyanarayana, D. R. K. Reddy, Bianchi type-III dark energy model in a Saez-Ballester scalar-tensor theory, International Journal of Theoretical Physics, 51, 2012, 2857-2862

[17]. T. Padmanabhan, and T. Roy Choudhury, A theoretician's analysis of the supernova data and the limitations in determining the nature of dark energy, Monthly Notices of the Royal Astronomical Society, 344, 2003, 823-834

[18]. L. Amendola, Acceleration at $\mathrm{z}>1$ ?, Monthly Notices of the Royal Astronomical Society, 342, 2003, 221-226

[19]. A. G. Reiss, et al., The farthest known supernova : support for an accelerating universe and a glimpse of the epoch of deceleration, Astrophysical Journal, 560, 2001, 49-71

[20]. H. Amirhashchi, A. Pradhan, B. Saha, An interacting two-fluid scenario for dark energy in an FRW universe, Chinese Physics Letters, 28, 2011a, 039801-4

[21]. A. Pradhan, H. Amirhashchi, Accelerating dark energy models in Bianchi type-V spacetime, Modern Physics Letters A 26, 2011, 2261-2275

[22]. A. Pradhan, A. K. Singh, H. Amirhashchi, A new class of Bianchi type-I cosmological models in scalar-tensor theory of gravitation and late time acceleration, International Journal of Theoretical Physics , 51, 2012, 3769-3786

[23]. A. Pradhan, A. K. Singh, D. S. Chouhan, Accelerating Bianchi type-V cosmology with perfect fluid and heat flow in SaezBallester theory, International Journal of Theoretical Physics, 52, 2013, 266-278

[24]. A. K. Yadav, Cosmological constant dominated transit universe from the early deceleration phase to the current acceleration phase in Bianchi-V spacetime, Chinese Physics Letters, 29, (7), 2012, 079801-5

[25]. K. S. Thorne, Primordial element formation, primordial magnetic fields, and the isotropy of the universe, Astrophysical Journal, $148,1967,51-68$

[26]. R. Kantowski, R. K. Sachs, Some spatially homogeneous anisotropic relativistic cosmological models, Journal of Mathematical Physics, 7(3), 1966, 443-446

[27]. J. Kristian, R. K. Sachs, Observations in cosmology, Astrophysical Journal, 143, 1966, 379-399

[28]. C. B. Collins, E. N Glass, D. A. Wilkinsons, Exact spatially homogeneous cosmologies, General Relativity and Gravitation, 12 (10), 1980, 805-823

[29]. M. A. H. MacCallum, A class of homogeneous cosmological models III : asymptotic behaviour, Communication in Mathematical Physics, 20, 1971, 57-84

[30]. A. G. Riess, et al., Observational evidence from supernovae for an accelerating universe and a cosmological constant, Astronomical Journal, 116, 1998, 1009-1038

[31]. S. Perlmutter, et al., Measurements of $\Omega$ and $\Lambda$ from 42 high-redshift supernovae, Astrophysical Journal, 517, 1999, 565-586

[32]. C. E. Cunha et al., Estimating the redshift distribution of photometric galaxy samples-II. Applications and tests of a new method, Monthly Notices of the Royal Astronomical Society, 396, 2009, 2379-2398 
[33]. C. B. Collins, Global structure of the Kantowski-Sachs cosmological models, Journal of Mathematical Physics, 18, 1977, 21162125

[34]. R. K. Knop, et al., New constraints on $\Omega_{M}, \Omega_{\Lambda}$ and w from an independent set of 11 high-redshift supernovae observed with the Hubble space telescope, Astrophysical Journal, 598, 2003, 102-137

[35]. M. Tegmark, et al., The three-dimensional power spectrum of galaxies from the sloan digital sky survey, Astrophysical Journal, $606,2004,702-740$

[36]. G. Hinshaw, et al., Five-year Wilkinson microwave anisotropy probe observations : data processing, sky maps, and basic results, Astrophysical Journal Supplement Series, 180, 2009, 225-245

[37]. E. Komatsu, et al., Five-year Wilkinson microwave anisotropy probe observations : cosmological interpretation, Astrophysical Journal Supplement Series, 180, 2009, 330-376 